Revisión

\title{
Quimioprevención del cáncer de mama
}

\author{
J. E. Alés Martínez
}

\section{Resumen}

La prevención farmacológica (quimioprevención) del cáncer de mama es el nuevo reto de la moderna oncología médica en el ámbito de este tumor, el más frecuente en la población femenina mundial. La extensión de los programas de cribado (detección precoz) y los avances en la farmacología terapéutica han conseguido que en la última década se registre un descenso constante en la mortalidad por esta enfermedad. Sin embargo, las tasas de incidencia continúan aumentando o cuando menos estabilizadas, lo cual hace, junto a la creciente proporción de mujeres en seguimiento después de completar el tratamiento adyuvante, que la carga médica y social del cáncer de mama continúe creciendo. Por ello, la consecución de un medicamento eficaz, seguro y tolerable que disminuya la incidencia del tumor es un objetivo altamente deseable. En la actualidad, una vez demostrado el potencial de la inhibición estrogénica con tamoxifeno para prevenir la aparición del cáncer de mama, se investiga activamente el papel de otros SERMS y algunos inhibidores de la aromatasa para conseguir un cociente riesgo-beneficio favorable en segmentos muy amplios de la población.

Palabras clave:

Quimioprevención. Cáncer de mama. Tamoxifeno. Exemestano. ExCel. Anastrozol.

Oncología, 2006; 29 (1):16-21

Programa de Investigación Clínica

Unidad de Oncología Médica

Hospital Ruber Internacional

Madrid

Recibido: 13.12 .05

Aceptado: 19.12.05 


\section{Summary}

The pharmacological prevention (chemoprevention) of breast cancer is the new challenge of modern medical oncology to deal with a kind of tumor that is the most frequent among the female population all over the world. The extension of the screening programs for early-stage detection and the advances in therapeutic pharmacology have achieved a continued reduction in breast cancer mortality in the last decade. However, the incidence rate continues increasing or is at least stable, what considered together with the increasing proportion of women in follow-up after completing the adjuvant treatment, leads to a continued increase of the medical and social burden of breast cancer. For this reason, the securing of an efficacious, safe and tolerated drug diminishing the tumor incidence is a very desirable objective. Presently, once demonstrated that tamoxifen-mediated estrogenic inhibition can prevent the appearance of breast cancer, the research of the role of other SERMs (Selective Estrogen Receptor Modulators) and aromatase inhibitors is very active, looking for products having a favorable risk-benefit ratio that will make then useful for wide groups of population.

Key words: Chemoprevention. Breast cancer. Tamoxifen. SERMs. Exemestane. ExCel. Anastrozol.

El cáncer de mama es un fenómeno de grandes proporciones a nivel mundial. Se estima que cada año se diagnostican alrededor de 1,2 millones de casos nuevos en el mundo y que unas 400.000 mujeres mueren por esta causa anualmente ${ }^{1}$. Las cifras españolas reflejan una incidencia aproximada de 15.979 casos y 6.000 muertes anuales ${ }^{2}$. De hecho, el cáncer de mama ocasiona la mayor mortalidad por cáncer en mujeres, seguido del colorrectal, estómago, páncreas y pulmón.

En los últimos 10 años los programas de detección precoz y los avances terapéuticos en el manejo de la enfermedad han logrado que la mortalidad por cáncer de mama descienda a un ritmo de un 1,4\% anual. Y ello a pesar de que la incidencia continúa aumentando a un ritmo de un 1-2\% anual. En la actualidad, la supervivencia del cáncer de mama a los 5 años del diagnóstico se eleva a un $78 \%$, frente al $70 \%$ que presentaba en el periodo 1985-1989.

No obstante estos avances, es indudable que si dispusiéramos de un tratamiento cómodo, bien tolerado y eficaz que disminuyera, de forma sustancial, la aparición del cáncer de mama, ello tendría una traducción inmediata en términos de bienestar social y mejora de la salud pública.

\section{Factores de riesgo}

La pertenencia al sexo femenino es el principal factor de riesgo para la aparición del cáncer de ma- ma y su desarrollo está íntimamente ligado a la exposición a estrógenos por parte del tejido glandular mamario. Este vínculo hormonal se ha detectado en numerosos estudios epidemiológicos ${ }^{3} \mathrm{y}$ se recoge, de forma indirecta, en varios componentes del principal algoritmo validado para predecir el riesgo de desarrollar cáncer de mama ${ }^{4-6}$. La manipulación del ambiente hormonal con fines preventivos adquiere mayor relevancia por el hallazgo de que la incidencia del cáncer de mama continúa aumentando, pero únicamente a expensas de los tumores con receptores hormonales positivos ${ }^{7}$.

\section{El bloqueo estrogénico puede prevenir el cáncer de mama}

Además de los hallazgos epidemiológicos, los datos en modelos animales ${ }^{8}$ y la constatación de que el tratamiento con tamoxifeno adyuvante en pacientes con cáncer de mama operable disminuía la incidencia de cáncer de mama contralateral ${ }^{9}$ justificaron el diseño y lanzamiento de varios ensayos de prevención del cáncer de mama con tamoxifeno a principios de la década de los noventa ${ }^{10-12}$.

El primer resultado positivo fue comunicado por el NSABP (National Surgical Adjuvant Breast and Bowel Project) en su estudio P-1, demostrando una reducción del $50 \%$ del riesgo de aparición de cáncer de mama en una población de riesgo elevado, seleccionada mediante el algoritmo de base epidemioló- 
gica de Gail ${ }^{10}$. Los ensayos realizados por el Royal Marsden y el grupo italiano se publicaron, inicialmente, como estudios negativos ${ }^{11,12}$. Más tarde, el ensayo IBIS-1 encontró resultados similares al estudio P-1, aunque el efecto preventivo fue algo menor. En una revisión reciente, que analiza en conjunto los datos actualizados de los estudios anteriores, se demuestra definitivamente que el tamoxifeno puede reducir el riesgo de aparición de cáncer de mama en mujeres con un riesgo superior al "normal"13.

Paralelamente, en el ensayo MORE, cuyo objetivo principal era demostrar la eficacia del tratamiento de la osteoporosis con raloxifeno (otro modulador selectivo de los receptores de estrógenos), la incidencia de tumores de mama se redujo drásticamente en las mujeres tratadas con raloxifeno en comparación con las tratadas con placebo ${ }^{14}$.

\section{Los límites del tamoxifeno}

El uso generalizado de tamoxifeno como agente preventivo se ha visto limitado porque se ha asociado con varios efectos secundarios potencialmente graves, destacando el cáncer de endometrio y los fenómenos de tromboembolismo venoso. Es fundamental que el agente elegido para la quimioprevención tenga un nivel de tolerancia y seguridad excelente. El grupo de trabajo al respecto de los Servicios de Prevención estadounidenses (U.S. Preventive Services Task Force) ha concluido que "los daños potenciales de la quimioprevención pueden superar los beneficios potenciales en mujeres que no tienen un riesgo alto de cáncer de mama". En el caso de las mujeres con riesgo alto, el informe declara que "el balance, entre beneficios y perjuicios, puede ser favorable en algunas mujeres de alto riesgo pero dependerá del riesgo de cáncer de mama, el riesgo potencial de efectos secundarios y las preferencias individuales de las pacientes" ${ }^{15}$.

\section{Quimioprevención con raloxifeno}

Se han diseñado varias estrategias con la intención de superar las limitaciones de tamoxifeno. En primer lugar, los prometedores resultados del estudio MORE dieron lugar al diseño y ejecución del estudio STAR (tamoxifeno frente a raloxifeno en mujeres postmenopáusicas con los mismos criterios de inclusión que el estudio P-1). Este ensayo, que ha completado recientemente la inclusión de sus 19.000 participantes nos dirá, a partir de 2006, si el balance riesgo/beneficio puede empujarse hacia el lado de los beneficios, gracias a una menor incidencia de nuevos casos de cáncer de endometrio y, tal vez, a una mayor eficacia preventiva de raloxifeno respecto a tamoxifeno. Sin embargo, no es probable que los problemas de tipo tromboembólico sean menores con raloxifeno que con tamoxifeno ${ }^{13,14}$. Se ha especulado que una pequeña dosis de antiagregantes plaquetarios puede contrarrestar este efecto protrombótico de los SERMS. Es posible que los resultados del estudio RUTH ${ }^{16}$, que compara raloxifeno con placebo en 10.101 mujeres con riesgo cardiovascular elevado, ayuden a despejar esta incógnita ya que el $50 \%$ de las pacientes incluidas tomaban aspirina a dosis bajas. Estos datos estarán disponibles también en 2006. Recientemente se han divulgado los resultados del estudio CORE, prolongación del ensayo MORE, donde se ha demostrado que los beneficios de raloxifeno en la prevención del cáncer de mama se mantienen después de 8 años de tratamiento $^{17}$

\section{Los inhibidores de la aromatasa no esteroideos}

Los inhibidores de la aromatasa han comenzado a investigarse como agentes preventivos debido a los favorables resultados con anastrozol, letrozol (inhibidores de la aromatasa de estructura no esteroidea) y exemestano (inhibidor esteroideo) en el tratamiento complementario del cáncer de mama operable.

En el estudio ATAC se demostró la superioridad de anastrozol en términos de eficacia y de seguridad respecto a tamoxifeno, incluyendo menos tumores de endometrio y menos episodios vasculares ${ }^{18}$. Además, la incidencia de nuevo cáncer de mama contralateral fue menor en las pacientes tratadas con anastrozol respecto a las tratadas con tamoxifeno, proporcionando el mismo tipo de evidencia que justificó los ensayos de quimioprevención con tamoxifeno. Por ello, se han puesto en marcha varios ensayos clínicos para comprobar si anastrozol mejora los resultados de tamoxifeno en mujeres con antecedentes de carcinoma de mama intraductal (NSABP B35, IBIS-II DCIS) o mujeres con riesgo elevado de cáncer de mama (IBIS-II). Los resultados de estos estudios estarán disponibles a partir de 2007, si 
se cumplen los plazos de inclusión previstos. Desafortunadamente, anastrozol no está exento de efectos indeseables. Su potente efecto antiestrogénico se ha asociado con un aumento de fracturas óseas en el ensayo ATAC. Este efecto secundario es especialmente preocupante, considerando que las mujeres posmenopáusicas ya tienen un riesgo mayor de fracturas osteoporóticas y el gran número de mujeres que se verían expuestas al fármaco en cualquier iniciativa poblacional de quimioprevención. Esto ha llevado a preconizar por algunos el uso simultáneo y profiláctico de inhibidores de la aromatasa y bifosfonatos para impedir la pérdida de masa ósea. Esta posible solución parece plausible, aunque faltan datos que confirmen su utilidad en el contexto de la quimioprevención. En cualquier caso, ello supone mayor complejidad de manejo y un gasto añadidos, que deben medirse con precaución en este contexto.

Letrozol es otro inhibidor no esteroideo de la aromatasa que ha demostrado su utilidad en el cáncer de mama avanzado y operable ${ }^{19,20}$. En el momento actual está en marcha el estudio WISE (Women with Increased Serum Estradiol) en el que se investiga el efecto de la toma de letrozol sobre el metabolismo óseo, la densidad mamaria y otros eventos en comparación con placebo, en mujeres postmenopáusicas con niveles altos de estradiol como criterio de inclusión principal. Presumiblemente, los beneficios y riesgos de letrozol serán similares a los anastrozol.

Exemestano es otro inhibidor de la aromatasa que, a diferencia de los anteriores, presenta una estructura y mecanismo de acción diferentes. Se trata de un agente esteroideo con una estructura similar al sustrato natural de la aromatasa, la androstendiona, que se une irreversiblemente al sitio catalítico de la enzima impidiendo su actividad hasta que se sintetiza de nuevo. Por este motivo se le considera un inhibidor suicida. En el estudio IES031, que comparó tamoxifeno con exemestano después de 2 a 3 años de tratamiento con tamoxifeno en mujeres posmenopáusicas ${ }^{21}$, quedó demostrada la superioridad de exemestano en cuanto a reducción de eventos mamarios: $32 \%$ menos que con tamoxifeno. Igualmente, la incidencia de cáncer de mama contralateral se redujo en más de un $50 \%$. El perfil de efectos secundarios fue generalmente favorable a exemestano con menos episodios tromboembólicos, de san- grado vaginal y de segundos tumores distintos del cáncer de mama, aunque con más artralgias y episodios de diarrea, casi siempre de carácter leve. En cuanto al hueso, hubo más episodios de osteoporosis y fracturas óseas en las mujeres tratadas con exemestano, aunque sin alcanzar significación estadística. Comunicaciones recientes sugieren un efecto óseo de exemestano similar al de otros inhibidores de la aromatasa ${ }^{22}$. El subestudio óseo incluido en el estudio MA27, que compara de forma directa anastrozol y exemestano en el tratamiento adyuvante del cáncer de mama con receptores hormonales positivos en mujeres postmenopáusicas resolverá, posiblemente, la cuestión de si existen diferencias significativas en su acción sobre el hueso entre los inhibidores de la aromatasa esteroideos y no esteroideos.

El buen perfil de eficacia y tolerancia de exemestano ha motivado su elección para un estudio de quimioprevención de gran importancia: el ensayo MAP-3/ExCel. En él se incluirán 4.560 mujeres postmenopáusicas de alto riesgo para cáncer de mama, seleccionadas en base a una puntuación superior a 1,67 en el test de Gail o antecedentes de lesiones mamarias indicativas de alto riesgo (hiperplasia ductal atípica, carcinoma lobulillar in situ, carcinoma intraductal operado con mastectomía simple). Las mujeres seleccionadas para el estudio, se asignarán aleatoriamente a una rama placebo o a otra con exemestano. El objetivo del estudio es disminuir la incidencia de cáncer de mama en la rama con exemestano en un $65 \%$ respecto a la rama con placebo. Este estudio tiene el interés añadido de que se incluirán 500 mujeres españolas por medio del Grupo Español de Investigación del Cáncer de Mama (GEICAM), con lo que se enriquecerá la homologabilidad de los resultados. En este sentido supone un hito fundamental en el desarrollo de la investigación en quimioprevención del cáncer de mama en España.

\section{Conclusiones}

La posibilidad de prevenir el cáncer de mama mediante la manipulación hormonal estrogénica es un hecho demostrado de forma definitiva. Aunque los efectos secundarios impiden su uso indiscriminado, existen grupos de mujeres que pueden benefi- 


\section{J. E. Alés Martínez}

ciarse ya hoy día del uso de tamoxifeno y disminuir así, de forma considerable, sus posibilidades de desarrollar cáncer de mama. En Estados Unidos se estima que más de 2 millones de mujeres podrían beneficiarse de la quimioprevención con tamoxifeno, tomando sólo en consideración aquellas con un beneficio neto estimado claro, fundamentalmente con edades menores de 50 años ${ }^{23}$. Los beneficios de tamoxifeno se han confirmado en un seguimiento a más largo plazo (7 años) de los resultados del estudio $\mathrm{P}^{24}$.

En la actualidad, existen diversos proyectos internacionales de investigación con la inclusión ya finalizada o en curso que, a partir de 2006, comenzarán a arrojar luz sobre si es posible mejorar el balance riesgo/beneficio de tamoxifeno. Sin embargo, quedan lagunas considerables sobre las que no hay iniciativas visibles a corto plazo, como son la prevención en mujeres premenopáusicas no elegibles para tamoxifeno o la prevención del cáncer de mama con receptores hormonales negativos. Las maniobras quimiopreventivas alternativas a la manipulación hormonal, como el uso de estatinas, no se han visto premiadas con el éxito hasta ahora ${ }^{25}$.

El estudio MAP-3 es un esfuerzo internacional colaborativo entre el Grupo de Ensayos Clínicos del Instituto Nacional del Cáncer de Canadá y el Grupo Español de Investigación del Cáncer de Mama que investiga la posibilidad de reducir la incidencia de cáncer de mama mediante la intervención con exemestano. En España, con una población potencial de más de un millón de mujeres posmenopaúsicas susceptibles de beneficiarse del tratamiento, podrían evitarse 2.000 o más casos nuevos al año de cáncer de mama, si se cumplen las expectativas del ensayo.

Correspondencia:

Dr. J. E. Alés Martínez

Programa de Investigación Clínica

Unidad de Oncología Médica

Hospital Ruber Internacional

La Masó, 38

E-28034 Madrid

\section{Bibliografía}

1. Ferlay J, Bray F, Pisani P, Parkin DM. GLOBOCAN 2000: Cancer Incidence, Mortality and Prevalence Worldwide, Version 1.0. IARC CancerBase No. 5. Lyon, IARCPress, 2001.

2. Incidence of cancer in Europe. www.eurocare.it. Actualización 2005.

3. Kelsey JL, Bernstein L. Epidemiology and prevention of breast cancer. Annu Rev Public Health. 1996; 17:47-67.

4. Gail MH, Brinton LA, Byar DP, Corle DK, Green SB, Schairer C, et al. Projecting individualized probabilities of developing breast cancer for white females who are being examined annually. J Natl Cancer Inst 1989; 81:1879-86.

5. Costantino JP, Gail MH, Pee D, Anderson S, Redmond $\mathrm{CK}$, Benichou J, et al. Validation studies for models projecting the risk of invasive and total breast cancer incidence. J Natl Cancer Inst 1999; 91:1541-8.

6. Anderson SJ, Ahnn S, Duff K. NSABP breast cancer prevention trial risk assessment program, Version 2. NSABP Biostatistical Center Technical Report. Pittsburgh (PA): Department of Biostatistics, University of Pittsburgh; August $14,1992$.

7. Jemal A, et al. Cancer Stadistics, 2003. CA Cancer J Clin. 2003 Jan-Feb;53(1):5-26.

8. Jordan VC. Effect of tamoxifen (ICI 46,474) on initiation and growth of DMBA-induced rat mammary carcinomata. Eur J Cancer 1976; 12:419-24.

9. Fisher B, Redmond C. New perspective on cancer of the contralateral breast: a marker for assessing tamoxifen as a preventive agent. J Natl Cancer Inst 1991; 83:1278-1280.

10. Fisher B, et al. Tamoxifen for prevention of breast cancer: report of the National Surgical Adjuvant Breast and Bowel Project P-1 Study. J Natl Cancer Inst 1998 Sep 16; 90(18):1371-88.

11. Powles T, et al. Interim analysis of the incidence of breast cancer in the Royal Marsden Hospital tamoxifen randomised chemoprevention trial. Lancet 1998 Jul 11; 352(9122):98-101.

12. Veronesi U, et al. Prevention of breast cancer with tamoxifen: preliminary findings from the Italian randomised trial among hysterectomised women. Italian Tamoxifen Prevention Study. Lancet 1998 Jul 11; 352(9122):93-7.

13. Cuzick J, et al. Overview of the main outcomes in breastcancer prevention trials. Lancet 2003 Jan 25; 361(9354):296-300.

14. Cauley JA, et al. Continued breast cancer risk reduction in postmenopausal women treated with raloxifene: 4-year results from the MORE trial. Multiple outcomes of raloxifene evaluation. Breast Cancer Res Treat 2001 Jan; 65(2):125-34.

15. U.S. Preventive Services Task Force. Chemoprevention of Breast Cancer: Recommendations and Rationale. Ann Intern Med 2002; 137(1):56-8.

16. Mosca L, et al. Design and methods of the Raloxifene Use 
for The Heart (RUTH) study. Am J Cardiol 2001; 88(4):392-5.

17. Martino JA, et al. Incidence of invasive breast cancer following 8 years of raloxifene therapy in postmenopausal women with osteoporosis: Results from the Continuing Outcomes Relevant to Evista (CORE) trial. 2004 ASCO Annual Meeting Abstract No: 1000.

18. Buzdar A, on behalf of the ATAC Trialists' Group. The ATAC ('Arimidex', Tamoxifen, Alone or in Combination) trial in post-menopausal women with early breast cancer updated efficacy results based on a median follow-up of 47 months. San Antonio Breast Cancer Symposium 2002. Abstract 13 .

19. Goss PE, et al. A randomized trial of letrozole in postmenopausal women after five years of tamoxifen therapy for early-stage breast cancer. N Engl J Med 2003; 349:1793802.

20. The Breast International Group (BIG) Collaborative Group. A comparison of Letrozole and Tamoxifen in postmenopausal women with early breast cancer. N Engl J Med 2005; 353:2747-57.
21. Coombes RC, et al. A randomized trial of exemestane after two to three years of tamoxifen therapy in postmenopausal women with primary breast cancer. N Engl J Med 2004; 350:1140-2.

22. Lonning PE, et al. Effects of exemestane administered for 2 years versus placebo on bone mineral density, bone biomarkers, and plasma lipids in patients with surgically resected early breast cáncer. J Clin Oncol. 2005;23:512637.

23. Freedman AN, et al. Estimates of the number of US women who could benefit from tamoxifen for breast cancer chemoprevention. J Natl Cancer Inst 2003; 95(7):52632.

24. Fisher B, et al. Tamoxifen for the prevention of breast cancer: current status of the National Surgical Adjuvant Breast and Bowel Project P-1 study. J Natl Cancer Inst. 2005 Nov 16;97(22):1652-62.

25. Bonovas S, Filioussi K, Tsavaris N, Sitaras NM. Use of statins and breast cancer: a meta-analysis of seven randomized clinical trials and nine observational studies. J Clin Oncol. 2005 Dec 1;23(34):8606-12. 